\title{
Isabelle Serça, Les coutures apparentes de la Recherche. Proust et la ponctuation
}

Paris, Honoré Champion, coll. « Recherches proustiennes », 2010, $276 \mathrm{p}$.

\section{Thomas Carrier-Lafleur \\ Université Laval et Université Paul-Valéry (Montpellier III)}

je file maintenant lavé délavé ouah voyons l'arabe appelle solaires les syllabes linguales dentales les autres lunaires l'expression chinoise lianxie veut dire lier en écrivant en avant l'enfant se débrouille d'abord avec ses accents multiples ou encore dans la flûte le si-bémol est la tonalité des adieux adieu o adieu je veux bien que ce soit en français si c'est vous qui le dites n'affirmons plus qu'il n'y a qu'une sorte de lutte il y en a deux la première sera louée de qui la comprendra l'autre est à condamner autrement dit tout ça est aussi vieux que le monde est ronde donc j'avais immédiatement deviné qu'il y avait une liaison entre ponctuation et procréation d'où leurs résistances clichés ponctuant miché leur journées à savoir qu'ils n'enregistrent que les points de rencontre avec leur image virgule tiret point virgule conclusion

Philippe Sollers, Paradis 
Le style de Proust a fait couler beaucoup d'encre. Plus particulièrement, la phrase proustienne compte maintenant une imposante bibliothèque critique qui tente, encore et encore, de l'expliquer. Certes, elle est longue, ardue, complexe, paradoxale. Mais ce n'est qu'un côté de la médaille. De l'autre, elle est poétique, sensuelle, corporelle, pointue, elle scrute le réel pour le radiographier. L'endroit double l'envers, et inversement. Avec ses parenthèses, ses tirets, ses incises, ses balbutiements et éternels retours déconstruisent le verbe par montage. C'est bien cela. La phrase proustienne est montage : elle fractionne le réel pour mieux l'étudier et, ensuite, elle le recompose, morceau par morceau, pour lui donner un sens nouveau. Toutefois, on ne peut pas dire que Proust fait un bon usage du français. Son écriture n'est pas classique. Elle n'est même pas conforme. Faites l'exercice: tapez, au hasard, n'importe quelle page d'À la recherche du temps perdu sur un logiciel de traitement de texte et demandez-lui une analyse textuelle. Maintenant, essayez de traduire le passage de la madeleine en latin... Alors, une question se pose, qui paraît toute simple: pourquoi Proust écrit-il de la sorte? À savoir : pourquoi Proust est-il Proust? Devant la circularité de telles interrogations, une réponse s'impose malgré tout. On la prendra chez Doubrovsky dans une conférence portant sur le début de la Recherche et qui mérite d'être relue. À travers une anecdote, il nous fait bien comprendre la démarche linguistique proustienne. Il dit donc ceci : "Il m'est arrivé récemment, à plusieurs reprises, eu égard aux infatigables progrès de la technique, qu'on me demande si j'avais besoin d'un word processor, d'une machine à traitement de texte, pour mon travail. Et, à chaque fois, la réponse qui m'est aussitôt venue à l'esprit, sinon aux lèvres, était: "la machine à traitement de 
texte, le word processor, c'est moi". Je ne vois pas de définition plus précise ni plus rigoureuse du travail de l'écrivain ${ }^{1}{ }^{\Perp}$. La réponse, qu'il faudra - on en convient - expliquer, est donc que Proust est son propre traitement de texte... En d'autres mots, Proust n'avait qu'une façon d'écrire la Recherche, celle que nous avons sous les yeux lorsqu'on en ouvre un volume, parce qu'il en avait littéralement besoin. Écrire autrement aurait été une altération du sujet écrivant. Or, les remarques de Gaëtan Picon sur le «chant proustien» sont toujours d'actualité. «Voici le courant qui ordonne les eaux contraires ; voici le rassemblement des forces, le juste élan. Voici le long, le doux, le prenant chant proustien, qui monte d'une grouillante pénombre, éclaire peu à peu les images de la vie comme, à travers les fentes des volets clos, le soleil éveille la chambre du dormeur ${ }^{2}$ ", avait-il écrit dans un élan lyrique notoire qui est lui-même une douce musique aux oreilles du lecteur proustien d'aujourd'hui. En somme, c'est la naissance d'une nouvelle voix. Avec un style décadent et des phrases alambiquées, on peut faire un roman génial. Mais il faut du talent. Plus encore, il faut une vision. En ce sens, Proust est un visionnaire.

\section{Langage, ponctuation... et pensée}

Toute grande œuvre littéraire crée un genre nouveau, ou brise un ancien. Dans le cas de Proust et de sa Recherche, c'est évident. En un mot, c'est un cas spécial. Walter Benjamin l'avait

\footnotetext{
1 Serge Doubrovsky, "Corps du texte/texte du corps», dans Autobiographiques: de Corneille à Sartre, Paris, Presses Universitaires de France, coll. «Perspectives Critiques », 1988 [1982], p. 44.

2 Gaëtan Picon, Lecture de Proust, Paris, Gallimard, coll. « Folio Essais », 1995 [1963], p. 43.
} 
remarqué, dès 1929, lorsqu'il écrivait que Proust, parmi les cas dits spéciaux, est l'un des plus insaisissables, " [à] commencer par sa structure, qui unit la fiction, les mémoires et le commentaire, jusqu'à la syntaxe avec ses phrases sans rivages (ce Nil du langage qui déborde ici, pour les fertiliser, sur les plaines de la vérité), tout ici échappe à la norme ${ }^{3} »$. C'est peu dire, la phrase proustienne est anormale, à commencer par sa ponctuation. C'est un fait. Mais peut-on l'expliquer? C'est à cette question que répond Isabelle Serça avec son ouvrage Les coutures apparentes de la Recherche : Proust et la ponctuation; livre qui, on peut en être sûr, deviendra un classique et une référence pour tous les proustiens. On avait déjà eu un avantgoût du travail d'I. Serça dans le récent recueil dirigé par Antoine Compagnon, Proust, la mémoire et la littérature, plus particulièrement avec son texte «Mouvement de la mémoire/mouvement de l'écriture : la figure de l'interpolation chez Proust». Y était étudié le rôle de la parenthèse proustienne comme figure d'interpolation, passant du microcosme de la phrase au macrocosme de l'œuvre. Ce questionnement sera repris et savamment développé avec Les coutures apparentes de la Recherche, pour notre plus grand plaisir. Or, le parti pris adopté par I. Serça est de taille et audacieux: il s'agit d'expliquer la Recherche proustienne, son esthétique, sa philosophie, sa vision du monde, à travers le prisme de sa ponctuation, plus encore grâce à l'usage unique qu'il fait de la parenthèse comme figure du discours, et des parenthèses - et des tirets, signes voisins, voire interchangeables - comme signes de ponctuation. Les enjeux soulevés sont majeurs. L'heure est grave. Il y a-t-il une analogie

3 Walter Benjamin, «L'image proustienne », dans OEuvres II, Paris, Gallimard, coll. « Folio Essais », 2007 [1929], p. 135. Nous soulignons. 
à établir entre le langage, sous sa forme écrite, et la pensée ? La langue exprime-t-elle vraiment la pensée ? Sans doute, mais il faut beaucoup de travail. C'est même une lutte. Lutte du langage contre lui-même. Un combat contre l'habitude. Comme dit Bergson (philosophe avec qui les affinités électives entre son œuvre et celle de Proust sont toujours à creuser), «[n]ous jugeons du talent d'un romancier à la puissance avec laquelle il tire du domaine public, où le langage les avait ainsi fait descendre, des sentiments et des idées auxquels il essaie de rendre, par une multiplicité de détails qui se juxtaposent, leur primitive et vivante individualité4 ${ }^{\text {}}$. L'habitude est l'ennemi ; le langage s'est fait prendre au piège. Mais c'est là que la littérature doit intervenir. Chez Proust, le romancier a pour devoir de redonner un sens profond au langage, qu'il ne soit pas seulement un vecteur social à la merci de la castration mimétique. Proust, avec son style unique, souhaite sortir la langue de la ritournelle de la "mêmeté ». La Recherche, en quête d'un usage original et sensible du langage, nous en offre une panoplie d'exemples. On en retiendra deux. D'une part, le passage où le héros-narrateur du roman nous livre sa vision du réel : «Ce que nous appelons la réalité est un certain rapport entre ces sensations et ces souvenirs qui nous entourent simultanément - rapport que supprime une simple vision cinématographique, laquelle s'éloigne par là d'autant plus du vrai qu'elle prétend se borner à lui - rapport unique que l'écrivain doit retrouver pour en enchaîner à jamais dans sa phrase les deux termes différents ${ }^{5}$ ». Ici, le cinématographe se

\footnotetext{
4 Henri Bergson, Essai sur les données immédiates de la conscience, Paris, Presses Universitaires de France, coll. «Quadrige: Grands Textes », 1889 [2011], p. 123-124.

5 Marcel Proust, Le Temps retrouvé, Paris, Gallimard, coll. «Folio Classique », 1990 [1927], p. 196.
} 
confond avec l'habitude. On lui demande du réel, du vrai à l'état pur, et, selon Proust, il ne nous donne que du même. Au contraire, la réalité perdue, c'est l'écrivain qui peut la retrouver. Sa phrase devient une sonde, car il la charge d'aller derrière les apparences, de l'autre côté du miroir. De l'autre côté du langage. Sinon, c'est peine perdue :

Si la réalité était cette espèce de déchet de l'expérience, à peu près identique pour chacun, parce que quand nous disons : un mauvais temps, une guerre, une station de voitures, un restaurant éclairé, un jardin en fleurs, tout le monde sait ce que nous voulons dire; si la réalité était cela, sans doute une sorte de film cinématographique de ces choses suffirait et le "style ", la «littérature» qui s'écarteraient de leurs simples données seraient un hors-d'œuvre artificiel ${ }^{6}$.

C'est dire que le roman proustien n'est pas un « hors-d'œuvre ». En effet, la question proustienne par excellence est la suivante : qu'est-ce que le réel ? Ou encore : comment parler du monde? De quelle manière peut-on être au monde? La réponse : par l'œuvre d'art. La Recherche incarne cet exutoire qui nous permet de toucher le monde. C'est toute la matérialité de l'œuvre littéraire. Elle rend tangible, palpable, le réel qui sans cesse paraissait nous fuir. Et qui dit œuvre littéraire dit langage. Qui dit langage dit ponctuation. Revenons aux Coutures apparentes de la Recherche, livre qui sans conteste, par l'analyse qu'I. Serça fait du rôle de la parenthèse en général et du style de l'auteur en particulier, détient une clé privilégiée pour accéder à l'univers proustien.

6 Ibid. 


\section{« Plaidoyer pro domo »}

S'il y avait encore des doutes quant à la pertinence d'étudier la parenthèse pour éclairer l'esthétique mise en avant par Proust dans sa Recherche, I. Serça les dissipe dans son plaidoyer d'ouverture. Ce qu'elle veut nous faire comprendre, c'est que la parenthèse proustienne est une figure majeure du roman, à l'égal de la métaphore et de la réminiscence. "Le parcours choisi traverse les contrées de la linguistique et de la littérature, en passant par les domaines de la grammaire, de la rhétorique, de la narratologie, de la poétique, de la théorie littéraire et enfin de l'esthétique» (p. 9), dit-elle. "[I]l redessine ainsi les frontières de la stylistique, point de jonction entre l'étude de la langue - la linguistique - et cet art qui emprunte la langue comme forme d'expression : la littérature » (p. 9).

La parenthèse est un point d'entrée important. Une fenêtre grande ouverte sur le style proustien. Par ricochet, I. Serça nous fait également remarquer que la ponctuation figure comme un thème du roman. Par exemple, pour expliquer les tics langagiers d'un personnage comme Françoise: «La duchesse doit être alliancée avec tout ça, dit Françoise [...]. Je ne sais plus qui qui [sic] m'a dit qu'un de ceux-là avait marié une cousine au duc. En tout cas c'est de la même "parenthèse" 7 ». Ou encore, quand le narrateur se renseigne presque maladivement sur Orianne, Saint-Loup, qui n'aime pas les interrogatoires, lui répond sèchement: «Mais oui! que de parenthèses ${ }^{8} !$ ». M. D'Argencourt, lors d'une conversation sur Mme de Guermantes, sera à son tour pris en flagrant délit : «Hier soir,

\footnotetext{
7 Id., Le côté de Guermantes, Paris, Gallimard, coll. « Folio Classique », 1988 [1920-1921], p. 16-17.

8 Ibid., p. 119.
} 
chez les Doudeauville, où, entre parenthèses, elle était splendide sous son diadème d'émeraudes, dans une grande robe rose à queue, elle avait d'un côté d'elle $\mathrm{M}$. Deschanel, de l'autre l'ambassadeur d'Allemagne ${ }^{9} »$. Parfois, grâce à ce procédé, l'on assiste à un curieux rapprochement entre le fond et la forme. Comme ici, lorsque Charlus converse avec le jeune narrateur à propos d'un souverain d'Europe et de ses problèmes de santé : «Les plus savants spécialistes de l'estomac le soignèrent sans résultat. Je l'amenai à un certain médecin (encore un être bien curieux, entre parenthèses, et sur lequel il y aurait beaucoup à dire $)^{10} »$. Finalement, le narrateur aussi se permet ce genre de remarques, qu'il adresse en plus au lecteur. L'écriture, dans sa matérialité, devient consciente d'elle-même : "Que cette parenthèse sur Mme de Forcheville, tandis que je descends les boulevards côte à côte avec M. de Charlus, m'autorise à une autre plus longue encore, mais utile pour décrire cette époque, sur les rapports de Mme Verdurin avec Brichot 11 ». C'est très clair, " [l]e procédé auquel Proust a souvent recours dans son livre est ainsi exhibé dans le texte même» (p.15). La parenthèse confirme la dimension autoréflexive de l'écriture proustienne.

De plus (et ce n'est une surprise pour personne), les parenthèses, en tant que signes de ponctuation, sont omniprésentes dans la Recherche. Évidemment, tout s'explique. Leur nombre élevé «va [...] de pair avec la longueur des phrases de Proust » (p. 16). Les critiques l'ont bien sûr évoqué, avec joie ou désarroi. D'autres, de Paul Morand à Jean Cocteau, ont même dit entendre les parenthèses dans la phrase, parlée,

\footnotetext{
${ }_{9}$ Ibid., p. 204.

10 Ibid., p. 281.

11 M. Proust, Le temps retrouvé, op. cit., p. 96.
} 
de Proust lorsqu'ils se fréquentaient. De surcroît, I. Serça nous informe que cette quantité impressionnante de parenthèses se retrouve tout autant dans la correspondance privée de Proust. Parfois, il arrive que Proust s'y perde. L'exemple relevé par I. Serça est splendide :

Telle grande courtisane dont l'évasure de la nuque a précisément la rondeur charmante de ces amphores où les Étrusques patients mirent tout leur idéal, tout leur rêve consolant de grâce, dont le coin de la lèvre est le même que dans ces vierges naïves de Luini (Bernardino) ou de Botticelli, que je préfère de beaucoup à celles de Raphaël - patatras, où en suis-je? - attends que je relise, ah! oui, telle courtisane, « dis-je », enferme dans les plis savamment ondulés de sa robe violette plus de charme que beaucoup de "salons »; surtout c'est plus moderne et très sincère. (Lettre de Proust à Robert Dreyfus, 10 septembre 1888 ; cité par I. Serça p. 18)

Étudier les manifestations puis le rôle des parenthèses chez Proust, c'est s'aventurer dans une cosmogonie. Un monde, littéralement, s'ouvre et se ferme sous nos yeux. Les parenthèses sont également synonymes de travail, de tâtonnement, d'essai et d'erreur. En un mot, de recherche.

Parallèlement, la parenthèse est emblématique de l'œuvre entière. L'œuvre en tant que tout. À savoir, «le rapprochement que l'on peut faire entre les parenthèses et la genèse de l'œuvre, qui s'est constituée par rajouts et corrections successives » (p. 18). Ce qui est fascinant, en effet, chez Proust, c'est son désir de toujours continuer. Jamais le romancier ne doit ou ne peut s'arrêter. Peut-être est-ce l'influence de Balzac ou de Zola. Quoique, à la différence de ces grandes fresques romanesques que forment Lacomédie humaine ou Les Rougont-Macquart, la Recherche ne s'est pas rédigée en continuité. Proust n'est pas un auteur linéaire - par 
là, on retrouve d'ailleurs sa vision du temps. On le sait maintenant, dans la chronologie de la rédaction, le début et la fin de l'œuvre sont contemporains. Du côté de chez Swann et Le temps retrouvé ont été écrits grosso modo au même moment. Le reste - et ce n'est pas rien - est une expansion par le milieu. Exactement comme les longues parenthèses qui habitent la phrase proustienne, c'est un foyer lumineux qui se propage. Littéralement, un univers en constante expansion. Malheureusement, on n'en connaîtra jamais les limites. Proust s'est éteint lorsqu'il corrigeait les épreuves de La prisonnière, cinquième des sept tomes de l'œuvre. Et quand on sait qu'au départ, des personnages comme Albertine n'étaient pas prévus dans la construction du roman, qui sait ce que Proust aurait pu $\mathrm{y}$ ajouter, par quelque effet de parenthèses...

\section{" Des raccrocs dans le bâti »}

Après avoir proposé une étude historique de la parenthèse, en miroir avec l'usage qu'en fait Proust, suivie d'une différenciation en la et les parenthèses et d'une étude de leurs différentes fonctions, allant de l'opposition entre les ajouts en étendue et en profondeur à la nature et au rôle de la digression, aux rapports entre juxtaposition et superposition, continuité et discontinuité, I. Serça explique, au cours de son huitième et dernier chapitre, la métaphore de son livre: la parenthèse comme couture apparente du roman proustien, comme raccroc dans le bâti du texte. La réflexion commence ici: les parenthèses proustiennes, remarquables et donc visibles, sont « un des moyens d'assurer la continuité au sein même de la discontinuité » (p.197). Elles tissent un texte au préalable décousu, mais bien souvent, leurs coutures sont apparentes. 
La parenthèse, c'est-à-dire «la figure de discours et le contenu textuel enserré» (p. 199), est un motif proustien capital qui, en plus, possède de nombreuses analogies avec d'autres procédés rois et maîtres de la Recherche, la réminiscence, en d'autres mots la mémoire involontaire, et la métaphore. Pourquoi ? Parce que la fonction des parenthèses est d'établir un rapport, de créer un liant. C'est la «fonction associative» (p. 201) de la parenthèse, dit I. Serça. Une métaphore culinaire peut nous éclairer, d'autant plus qu'elle se trouve chez Proust. Dans Le temps retrouvé, après avoir comparé son œuvre future à une cathédrale, un temple et une robe, le narrateur enchaîne avec cette curieuse comparaison. La théorie et l'esthétique du roman à venir sont maintenant associées à la cuisine de Françoise, à travers un plat bien précis : «ne ferais-je pas mon livre de la façon que Françoise faisait ce bœuf mode [...] et dont tant de morceaux de viande ajoutés et choisis enrichissaient la gelée ${ }^{12} »$. L'œuvre littéraire est faite de fragments, de morceaux épars, voire volatiles. Le but du romancier est de trouver un agencement, une manière de les faire tenir. C'est une question de composition et de mise en scène. Plusieurs dispositifs seront convoqués. On l'a dit: la mémoire involontaire, les métaphores et les parenthèses. Du bœuf mode, les parenthèses sont la gelée. Elles unissent les morceaux que l'auteur aura retenus pour son œuvre. Entre le narrateur, Françoise et Michel-Ange, pas de différences: « comme elle attachait une importance extrême à la qualité intrinsèque des matériaux qui devaient entrer dans la fabrication de son œuvre, elle allait elle-même aux Halles se faire donner les plus beaux carrés de romsteck, de jarret de

12 Marcel Proust, Le Temps retrouvé, op. cit., p. 340. 
bœuf, de pied de veau, comme Michel-Ange passant huit mois dans les montagnes de Carrare à choisir les blocs de marbre les plus parfaits pour le monument de Jules II $^{13} »$. Ils sont tous contemporains.

Ce qu'il faut retenir de cet exercice, c'est la folle ambition de Proust de vouloir s'approprier tous les points de vue à la fois. L'œuvre littéraire, telle une armée en guerre, doit être un Argus aux cent yeux. La vérité, à savoir le temps et le réel à l'état pur, n'est rendue possible que par cette multiplicité. Notre vision des choses est toujours fragmentaire, il nous faut alors trouver un appareil qui serait en mesure de collecter et de joindre tous les points de vue possibles. Évidemment, il s'agit du roman. Or, dans le texte proustien, remarque avec justesse I. Serça, la parenthèse est un mécanisme privilégié de l'appareil. La parenthèse est une transversale entre les différents morceaux de l'œuvre littéraire qui sont tous un monde. Dans le roman, ils peuvent tous coexister. L'esthétique de la parenthèse, souligne I. Serça, est aussi exemplifiée par le passage célèbre où le jeune narrateur se rend pour la première fois à Balbec. Il est en train et constate le lever de soleil. Magnifique. Mais, tout à coup, le train change de direction et «la scène matinale fut remplacée dans le cadre de la fenêtre par un village nocturne aux toits bleus de clair de lune ${ }^{14} »$. Le narrateur doit changer de fenêtre, pour retrouver son coucher de soleil. Il est devant le morcellement du monde, illustré ici par les différentes fenêtres d'un train en marche, qui sont toutes une monade sur un univers particulier. Du coup, il a une idée : «je passais mon temps à courir d'une fenêtre à l'autre pour rapprocher, pour

13 Id., À l'ombre des jeunes filles en fleurs, Paris, Gallimard, coll. «Folio Classique », 1988 [1919], p. 17.

14 Ibid., p. 223-224. 
rentoiler les fragments intermittents et opposites de mon beau matin écarlate et versatile et en avoir une vue totale et un tableau continu 15 ». I. Serça en conclut que " [l]a chute de ce passage offre un florilège de termes éminemment proustiens et illustre à merveille cette vision fragmentaire, donnant à voir les intermittences du cœur d'un moi versatile à la recherche de la continuité. Toute l'activité du Je consiste en effet à créer des liens dans un mouvement de navette d'un point de vue à un autre » (p. 203). La parenthèse, comprise de la sorte, devient un vecteur de points de vue, voire de mondes différents qui pourront ainsi s'unifier en nous, par le biais de l'œuvre d'art issue de notre moi profond. Il suffit « d'assembler les morceaux du puzzle» (p. 203). Encore un casse-tête, signé Proust.

Les parenthèses et « [l]es transversales — à l'image de ce mouvement de navette - sont précisément les liens que le texte tisse entre ces fragments "opposites" pour les "rentoiler" » (p. 204). Elles assurent la continuité, à même la discontinuité. Les parenthèses permettent au romancier d'acquérir une multiplicité de points de vue. Un regard neuf sur le monde. On remarque un paradoxe, mais riche de sens: les parenthèses proustiennes, "[é]léments tout à la fois centripètes et centrifuges » (p.205), fragmentent le texte en général et la phrase en particulier (et y insérant, parfois de façon violente, un élément étranger, souvent sous le couvert de la métaphore ou de la comparaison); elles brisent donc l'unité ; mais, du coup, c'est pour mieux y reconstruire et y replacer une unité nouvelle, un autre regard qui rejoint volontiers l'esthétique de l'œuvre entière. L'art du roman. Les parenthèses sont là pour ouvrir le texte, non pas pour le refermer sur lui-même. C'est

15 Ibid., p. 224. 
pourquoi la phrase de Proust ne doit pas être perçue comme compliquée, fine, voire précieuse. Son intérêt, somme toute, n'est pas là. L'écriture proustienne est une écriture qui se manifeste. Elle est consciente d'elle-même. Dit autrement, Proust, dans ses phrases, est toujours à la recherche. Il se fait enquêteur. La parenthèse est pour lui un outil privilégié d'exploration du réel. Du réel dans sa totalité et dans sa grandeur. Pas dans le détail. C'est l'opposition toute proustienne entre le télescope - outil privilégié par le héros, futur romancier - et le microscope - lui, à proscrire. Le narrateur ne s'en rend compte qu'en fin de piste ${ }^{16}$, alors que pour le lecteur, c'est une prémisse des plus essentielles, d'où l'importance de l'ouvrage d'I. Serça.

\section{"La seule manière de défendre la langue, c'est de l'attaquer »}

«Les parenthèses de Proust sont ainsi des points de suture du texte : elles rapprochent - en tirant parfois sur les bords - des éléments différents pour les faire tenir ensemble. Elles sertissent les pièces de verre coloré du vitrail : ces motifs ne coïncident pas bord à bord mais, pris dans le même plomb, ils forment alors une

\footnotetext{
16 C'est un des beaux passages du roman, où l'on entend nettement la voix de Proust derrière celle de son narrateur : «Bientôt je pus montrer quelques esquisses. Personne n'y comprit rien. Même ceux qui furent favorables à ma perception des vérités que je voulais ensuite graver dans le temple, me félicitèrent de les avoir découvertes au " microscope », quand je m'étais au contraire servi d'un télescope pour apercevoir des choses, très petites en effet, mais parce qu'elles étaient situées à une grande distance, et qui étaient chacune un monde. Là où je cherchais les grandes lois, on m'appelait fouilleur de détails. D'ailleurs, à quoi bon faisais-je cela ? J'avais eu de la facilité, jeune, et Bergotte avait trouvé mes pages de collégien "parfaites". Mais au lieu de travailler j'avais vécu dans la paresse, dans la dissipation des plaisirs, dans la maladie, les soins, les manies, et j'entreprenais mon ouvrage à la veille de mourir, sans rien savoir de mon métier » (Le Temps retrouvé, op. cit., p. 346).
} 
image unie bien qu'elle projette des reflets multiples, étant faite de morceaux» (p. 213). C'est ce qui fait aussi de la Recherche une œuvre rhapsodique, faite de fragments - fragments qui permettent un va-et-vient constant entre le microcosme de la phrase et le macrocosme du roman, en quête de points de vue, compris en tant que machine à explorer le réel. La ponctuation est élevée au niveau de l'esthétique, puisque Proust use des parenthèses d'une façon qu'il faudrait bien se résoudre à nommer philosophique. $\mathrm{Si}$, comme le dit le narrateur proustien, son roman doit être conçu comme une robe, les parenthèses sont là pour à la fois coudre le texte, le resserrer, l'unir, mais au final elles le fragmentent tout autant, à travers ces morceaux de réel à l'état pur qu'il ne faut pas seulement ramener à l'état de simples digressions, aussi brillantes soient-elles. Les parenthèses sont des raccrocs dans le bâti du texte du Proust, nous offrant un tissu textuel cousu, décousu, puis recousu, finalement offert au lecteur afin qu'il confectionne son propre vêtement. I. Serça, avec cette théorie, ne nous présente pas l'image d'un Proust snobinard, dandy, obsédé par l'observation et seulement par l'observation. Heureusement. À l'inverse, c'est un Proust ouvrier, qui écrit sans compter, qui se relit, qui ajoute, découpe, recoupe, taille et retaille. "Proust: CEuvre comme faite par une Couturière ${ }^{17}$ », disait Barthes dans un éclair de génie.

Secrètement, toutes ces questions dessinent un enjeu majeur : l'usage de la langue par les écrivains. Nous en avons fait mention plus haut: un des grands combats menés par Proust a été celui contre l'habitude. Surtout dans une œuvre aussi personnelle que la Recherche, qu'il ne faut pas hésiter à

17 Roland Barthes, La préparation du roman I et II. Cours et séminaires au Collège de France (1978-1979 et 1979-1980), Paris, Seuil/IMEC, coll. « Traces écrites », 2003, p. 203. 
qualifier d'autofiction avant la lettre. Avec l'écriture, le travers sur le langage et le combat avec la langue, on peut finalement surmonter notre moi social, dépasser les conventions et retrouver notre moi profond. Encore une fois, la parenthèse est bien plus qu'un signe typographique innocent. Elle devient un instrument ou une arme. Une antichambre de la création. «Miroir d'une écriture qui se réfléchit dans le cours du texte, la parenthèse est le lieu de prédilection d'un Je qui cherche à se saisir lui-même dans un mouvement de réflexion. La parenthèse est ainsi par essence le mode d'expression de la première personne, la figure même de l'introspection » (p. 221). C'est ainsi que l'on gagne à relire ce morceau notoire de la correspondance de Proust avec Mme Straus :

Chaque écrivain est obligé de se faire sa langue, comme chaque violoniste est obligé de se faire son "son». [...] La seule manière de défendre la langue, c'est de l'attaquer, mais oui, Madame Straus! Parce que son unité n'est faite que de contraires neutralisés, d'une immobilité apparente qui cache une vie vertigineuse et perpétuelle. Car on ne "tient», on ne fait bonne figure, auprès des écrivains d'autrefois qu'à condition d'avoir cherché à écrire tout autrement. Et quand on veut défendre la langue française, en réalité on écrit tout le contraire du français classique ${ }^{18}$.

Les parenthèses ont permis à Proust de trouver son "son ». La façon d'écrire du romancier, comme le système conceptuel pour le philosophe, n'est que le reflet que sa subjectivité. Le travail sur le dit et le dire n'est qu'une altération - mais créatrice de la syntaxe. La linguistique devient esthétique. De la ponctuation en général, on arrive au sommet du style de Proust en particulier.

18 Marcel Proust, Correspondance avec madame Straus, Paris, Plon, coll. « Le livre de poche », 1974 [1936], p. 110-111. 